\title{
A Peptide Containing T-Cell Epitopes of Chlamydia trachomatis Recombinant MOMP Induces Systemic and Mucosal Antibody Responses in Mice
}

\author{
Murtada A. Taha, Shree R. Singh, Kara Hulett, Shreekumar R. Pillai, Ronald Agee, Vida A. Dennis ${ }^{*}$ \\ Center for NanoBiotechnology and Life Science Research, Alabama State University, Montgomery, USA. \\ Email: *vdennis@alasu.edu
}

Received March $4^{\text {th }}, 2011$; revised May 12 $2^{\text {th }}, 2011$; accepted May $29^{\text {th }}, 2011$.

\begin{abstract}
We reported that intranasal immunization of mice with recombinant major outer membrane protein (MOMP) of Chlamydia trachomatis genetically fused with modified cholera toxin elicited mucosal and systemic antibody responses, but with inefficient protective mechanisms for complete protection of mice against a homologous $C$. trachomtis challenged infection. To begin to identify specific immunogenic MOMP regions to pursue as vaccine candidates, herein we selected small gene fragments of the MOMP gene containing T-cell epitopes (278 - 370 aa) and generated a rMOMP peptide (rMOMP-278). rMOMP and rMOMP-278 proteins were cloned, expressed, and their purities and specificities confirmed by SDS-PAGE and Western blot, respectively. We tested the immunogenicity of rMOMP-278 as compared to the parent rMOMP in mice. Mice were immunized intramuscularly with purified rMOMP proteins; total-and isotype- (IgA, $\operatorname{Ig} G 1, \operatorname{IgG2a}$, and $\operatorname{Ig} G 2 b)$ specific antibodies in sera and vaginal washes were measured by ELISA. Immunized mice developed antigen-specific total antibodies in a kinetic fashion, with responses being higher to rMOMP than rMOMP278. However, antigen-specific isotype antibodies were detected in the order of $\operatorname{Ig} G 2 b>\operatorname{Ig} G 2 a>\operatorname{Ig} G 1$ for rMOMP278 , indicating more of a mixed $T h_{1} / T h_{2}$ response. Contrastingly, antibody responses to rMOMP was more of a predominant $T h_{2}$ response in the order of $\operatorname{IgG1}>\operatorname{IgG2b}>\operatorname{IgG2a}$. Our data are evidence to suggest that $r M O M P-278$ is immunogenic by its ability to evoke systemic and mucosal immune responses with a $T_{1}$ bias in mice, and therefore may be an attractive peptide alternative to full MOMP as a vaccine candidate against $C$. trachomatis genital tract infection.
\end{abstract}

Keywords: Bacteria, rMOMP, Mucosal Vaccine, Antibody

\section{Introduction}

Chlamydia trachomatis, an obligate intracellular Gramnegative bacterium, is the leading cause of bacterial sexually transmitted infections (STIs) in the world. Genital tract infection with $C$. trachomatis, poses a significant risk in women, often leading to chronic abdominal pain, pelvic inflammatory disease (PID), fallopian tube scarring, ectopic pregnancy, and infertility. Although effective antimicrobials are available for Chlamydia, this approach has been unsuccessful in curtailing the spread of infections because the latter are frequently asymptomatic. Such insidious infections make diagnosis and application of antibiotic therapy late and ineffective. Therefore, development of a safe efficacious prophylactic mucosal vaccine for $C$. trachomatis is the key to re- duce its transmission. However, to date, no efficacious vaccine against this human pathogen is available.

Development of a vaccine against $C$. trachomatis is challenging, in part, because of poor understanding of the regulation of the immune response in the female genital tract, the lack of adjuvant that target vaccines to the genital mucosa and our limited knowledge of which $C$. trachomatis antigens induce protective immune responses [1-3]. Thus, recent vaccine development efforts are now being targeted towards subunit or multimeric vaccines.

Prominent amongst these subunit vaccine is the immunodominant MOMP (major outer membrane protein) of $C$. trachomatis which is now being evaluated for its prophylactic potential in several animal models [4-14]. 
MOMP is surface accessible and highly immunogenic, eliciting both neutralizing antibodies and T-cell responses. The rMOMP gene consists of an 1182 base pair open reading frame that encodes a 394 amino acid long protein, which contains 8 cysteine residues [15]. MOMP constitutes approximately $60 \%$ of the total protein mass of the bacterial outer membrane, encoding for nine distinct amino acid sequences or regions [3]. Five of these regions are highly conserved across $C$. trachomatis serovars while the remaining four regions are known as variable domains (VD). The domains designated VD-1, VD-2, and VD-4 are exposed on the surface of the organism and contain documented epitopes.

Studies have shown that MOMP delivered via Vibrio cholerae ghosts $[2,16]$, linked to the modified cholera toxin [8] or complexed with immune stimulating complexes [17] elicits mucosal immune responses, and protection from C. trachomatis infections. Nevertheless, a successful $C$. trachomatis vaccine would need to induce a systemic and mucosal $\mathrm{Th}_{1}$ immune response, to deal with $C$. trachomatis as an intracellular pathogen, plus a local mucosal IgA response to reduce bacterial shedding and the resulting spread of infection to sexual partners of infected individuals [15]. Thus continuous efforts are ongoing to develop a vaccine that can ultimately induce sterilizing immunity against a $C$. trachomatis infection.

T-cell epitopes of the MOMP of C. trachomatis may prove to be more effective vaccine candidates against Chlamydia than the entire full length MOMP. The extreme hydrophobicity of MOMP may create problems in the use of the whole MOMP molecule in vaccines. In addition, immunization with an intact protein, at times, can lead to suppression of immune responses to the same or other antigens [18]. In humans, MOMP elicits C. trachomatis neutralizing antibodies that recognize its surface-exposed variable segments (VSs), where amino acid sequences vary among serovars [19]. Previously we reported that intranasal immunization with the recombinant MOMP (rMOMP) of C. trachomatis genetically fused with modified cholera toxin elicited mucosal and systemic antibody responses, which in part protected mice against a homologous challenged infection [8]. However, this vaccine formulation failed in the sense that it did not induce protective mechanisms to provide complete protection of mice against a $C$. trachomatis genital tract infection. In pursuit of MOMP regions that may contribute, and provide protective immunity in mice, herein we generated a peptide of C. trachomatis MOMP [small gene fragments containing T-cell-epitopes (278-370 aa) [18, 20,21], designated rMOMP-278)] and tested its immunogenicity, as compared to that of the parent rMOMP in mice, without the use of an adjuvant delivery system.
Both rMOMP and rMOMP-278 were amplified by PCR, cloned into pET-32 vector, expressed and purified. $\mathrm{BALB} / \mathrm{c}$ mice were immunized with purified full MOMP or with MOMP-278 and systemic and mucosal antibody responses were determined by ELISA in serum and vaginal wash samples. Our results are presented in this manuscript.

\section{Materials and Methods}

\subsection{Bacterial Strains, Cells, and Culture of C. trachomatis MoPn Nigg II}

E. coli Novablue and E. coli BL21 (DE3) cells were purchased from Novagen (Novagen, Madison, WI) and used for cloning and expression experiments, respectively. Cells were grown at $37^{\circ} \mathrm{C}$ in Luria Bertani (LB) medium containing ampicillin for cloning; clones used for expression were grown at $30^{\circ} \mathrm{C}$ in the same medium.

C. trachomatis MoPn NiggII was purchased from ATCC (Manassas, VA, ATCC \#VR-123). Stock preparations of C. trachomatis elementary bodies (EBs) were produced by propagation in HeLa 229 cells as described previously [8]. All stocks were titrated on HeLa cell monolayers using the inclusion forming unit (IFU) assay, purified over renograffin gradients and stored at $-70^{\circ} \mathrm{C}$ until they were used.

\subsection{Cloning of pET-MOMP and pET-MOMP-278}

Polymerase Chain Reaction (PCR) was carried out to amplify antigenic regions of $C$. trachomatis MOMP gene using custom primers and C. trachomatis MoPn NiggII gene as a template. The primers used to amplify the MOMP gene fragment (corresponding to nucleotides 2221379 [8]) and MOMP-278 region (corresponding to nucleotides 1003-1283) are mompF (GTCGTTCCATGGTTCCTCCTTGCATGCATGCTCTGC), mompR (AAGTAAGTCGACGGAACTGAGCATTTACGTGAAGC), mopnF (GTCGTTCCATGGGACTGAATATGTTCACTCC), and mopnR (AAGTAAGTCGACTCTCAACAGTAACTGC) which contain $\mathrm{Sal}$ I and $\mathrm{NcoI}$ restriction sites. DNA was denatured at $94^{\circ} \mathrm{C}$ for $5 \mathrm{~min}$ and PCR amplification was performed for 34 cycles at $94^{\circ} \mathrm{C}$ for 15 $\mathrm{sec}, 55^{\circ} \mathrm{C}$ for $30 \mathrm{sec}$, and $72^{\circ} \mathrm{C}$ for $1 \mathrm{~min}$. The DNA extension was achieved at $94^{\circ} \mathrm{C}$ for $15 \mathrm{sec}, 55^{\circ} \mathrm{C}$ for $30 \mathrm{sec}$, and $72^{\circ} \mathrm{C}$ for $1 \mathrm{~min}$. Qiagen gel extraction and plasmid midi kits (Qiagen Inc., Valencia, CA) were used to purify PCR amplified fragments and plasmid DNA. Purified plasmid and PCR amplified insert DNA were digested with NcoI and SalI restriction enzymes (Invitrogen, Carlsbad, CA) following the manufacturer's protocol. Vector DNA was treated with CIAP to dephosphorylate 
the linearized vector. Purified restriction enzyme digests of DNA were used for ligation. The vector and insert were ligated and the ligation mix was then used to transform E. coli Novablue cells following the manufacturer's transformation protocol. Recombinant clones were purified using Qiagen plasmid midi kits (Qiagen Inc.). Purified plasmid DNA clones were analyzed by restriction enzyme digestion and the sequences between the thioredoxin and $\mathrm{Tt} 7$ were confirmed by DNA sequencing (Auburn University Genomics and Sequencing laboratory, Auburn, AL) using Stag, T7 and MOMP primers. The purified $\mathrm{pET}-\mathrm{MOMP}$ and $\mathrm{pET}$-MOMP-278 vectors were used to transform E. coli BL21 (DE3) expression host cells (containing inducible T7 RNA polymerase) and used for all protein expression experiments.

\subsection{Protein Expression, Preparation and Purification of Soluble Protein}

E. coli BL21 (DE3) cells containing the pET-MOMP, and pET-MOMP-278 vector were grown in LB broth and induced by isopropyl- $\beta$-D-thiogalactoside (IPTG) and purified using His-Bind Columns (Novagen, Madison, WI) as already described by us [8]. The resulting protein was analyzed on SDS-PAGE and the protein was shown to have one band which corresponded to the recombinant protein and was termed rMOMP or rMOMP-278 and used in all future studies. The recombinant clones were also analyzed by DNA sequencing.

\subsection{SDS-PAGE and Western Blotting}

Cytoplasmic extract, IB soluble protein, and the insoluble pellet were all run on $10 \%-20 \%$ pre-cast gradient gel (Bio-Rad Laboratories, Hercules, CA) to perform protein analysis as already described [8]. Briefly, proteins were transferred onto a PVDF membrane probed with antiMOMP antibodies (Fitzgerald Industries, Concord, MA) followed by the appropriate HRP-conjugated secondary antibody (Sigma, St. Louis, MO). The bound antibody was detected using a chemiluminescent substrate (ECL+ Amersham Pharmacia Biotech, Piscataway, NJ). Chemiluminescent of PVDF membranes was performed using the Alfa Innotech gel documentation system (Alfa Innotech, San Leandro, CA).

\subsection{Immunization Studies}

Four- to six-week-old female BALB/c mice (The Jackson Laboratory, Bar Harbor, ME) were used for this study. All mice protocols were approved by the IACUC at Alabama State University. Animals were housed at an IACUC approved animal facility under a standard day and night cycle, and provided food and water ad libitum. Three groups of mice containing six mice per group were immunized intramuscularly (i.m) using purified rMOMP, rMOMP-278, or PBS as a control. Mice in groups 1 and 2 were immunized with $50 \mu \mathrm{g}$ of $\mathrm{rMOMP}(1 \mu \mathrm{g} / \mu \mathrm{L}$ in PBS) and $50 \mu \mathrm{g}$ of rMOMP-278 ( $1 \mu \mathrm{g} / \mu \mathrm{L}$ in PBS), respectively. Mice in group 3 served as negative control, and were immunized with $50 \mu \mathrm{L}$ of PBS. Serum and vaginal wash samples from all three groups of mice were collected on days $0,7,14$, and 21 pre and post-immunization to determine antibody responses. Vaginal wash samples were collected by flushing the vagina with 50 $\mu \mathrm{L}$ of sterile PBS.

\subsection{Determination of Antibody Responses}

ELISA was employed to determine antibody responses in serially collected serum and vaginal wash samples from immunized and control mice. To analyze the anti-MOMP antibody response, $100 \mu \mathrm{L}$ of UV-inactivated $C$. trachomatis in PBS was added to the appropriate wells of an ELISA plate. In addition to the use of $C$. trachomatis as an antigen, rMOMP and rMOMP-278 purified proteins were also used as antigens. In order to analyze the serum and vaginal wash samples from group one (immunized with rMOMP) an ELISA plate was coated with the rMOMP protein at $1 \mu \mathrm{g} / \mathrm{mL}$ and for group two (immunized with rMOMP-278) an ELISA plate was coated with the rMOMP-278 protein at $1 \mu \mathrm{g} / \mathrm{mL}$. For antibody concentrations, serial dilutions $(1: 250,1: 500$, and 1:1000) of the samples were made in antibody buffer ( $2 \%$ nonfat dry milk in PBS with $0.05 \%$ Tween 20), and ELISAs were performed essentially as already reported by us [8] using HRP-conjugated goat anti-mouse Ig (1:2000) (Southern Biotech Associates (SBA), Birmingham, AL) and TMB as substrate. Normal mouse serum served as the negative control. Absorbance was read at $450 \mathrm{~nm}$ using a Tecan plate reader. Readings at least three times that of the negative control were considered positive and only readings with an absorbance value of $\geq 0.1$ were considered significant. For both serum and vaginal wash samples, a dilution of 1:250 gave the highest antibody responses and thus was used for all analyses in this study.

Isotype determination kits (SBA) were used for antibody isotype determination and as described above, plates were coated with rMOMP (rMOMP or rMOMP278) or C. trachomatis and serial dilution of serum and vaginal wash samples were added to each well. Goat anti-mouse HRP-conjugated secondary antibodies to IgG1, IgA, IgG2a, and IgG2b were diluted $(1: 2000)$ and $100 \mu \mathrm{L}$ of each was added to the appropriate wells. The reaction was then developed, and absorbance was read at $450 \mathrm{~nm}$. Readings at least three times that of the negative control were considered positive. 


\subsection{Statistical Analysis}

All the data are expressed as means \pm SD of samples run in triplicate. Data were analyzed by ANOVA single factor using different between means of two treatments. $P$ values $\leq 0.05$ were considered significant.

\section{Results}

\subsection{Cloning of MOMP T-Cell Epitopes}

In order to generate the desired MOMP T-cell epitope, the MOMP gene was isolated from C. trachomatis MoPn NigII purified EBs and used as a template. Using primers mopn278 and mopn370, the T-cell epitope corresponding to the $1003-1283$ regions of MOMP was amplified by PCR using template DNA which resulted in a fragment of approximately $300 \mathrm{bp}$ (data not shown). Similarly, the full length MOMP gene corresponding to 222-1379 of the MOMP gene was amplified by PCR and used to generate the MOMP vector resulting in a fragment of approximately $1157 \mathrm{bp}$ (data not shown). Purified plasmid DNA clones were further confirmed by DNA sequencing.

\subsection{Solubilization, Purification and Characterization of the Proteins}

Since most of the rMOMP and rMOMP-278 proteins were present in the inclusion bodies, proteins were solubilized and along with the cytoplasmic extract and insoluble protein fractions (pellet) were analyzed by SDSPAGE. As shown in Figure 1, a band with an apparent molecular weight of $\sim 60 \mathrm{kDa}$ corresponding to rMOMP (Figure 1(a), lane 5) and a band of $\sim 31 \mathrm{kDa}$ corresponding to rMOMP-278 (Figure 1(b), lane 5) was observed after purification. Western blot analyses using anti-chlamydial polyclonal antibodies further confirmed the specificities of the respective purified rMOMP (Figure 1(c), lane 2) and rMOMP-278 (Figure 1(d), lane 2) proteins of $60 \mathrm{kDa}$ and $31 \mathrm{kDa}$, respectively. The rMOMP $60 \mathrm{kDa}$ and $31 \mathrm{kDa}$ proteins were also observed in the IB fractions as well (Figures 1(c) and 1(d), lane 2).

\subsection{Antibody Responses}

To study the immune response of rMOMP and MOMP278 , three groups of BALB/c mice were immunized intramuscularly (i.m) with rMOMP, rMOMP-278, or with PBS as a negative control. Serum and vaginal wash samples were collected from mice on days $0,7,14$ and 21 pre-and post-immunizations and analyzed for the presence of rMOMP-specific antibodies. Serum antibody responses were essentially negligible in all groups of mice prior to immunization (day 0 ). Intramuscular ad- ministration to mice of rMOMP or rMOMP-278 resulted in the production of serum and vaginal wash total Ig antibodies as early as day 7 after the first immunization when compared to that of day 0 (Figures 2(a) and 2(b)). Serum and vaginal wash Ig concentrations were further significantly $(P<0.05)$ elevated after the second and third immunizations in mice immunized with rMOMP. However, serum Ig concentrations in mice immunized with rMOMP-278 remained relatively unchanged between days 7 and 14 but increased markedly at day 21, suggesting a delay production of Ig in them. No further increases in vaginal wash Ig from rMOMP-278 immunized mice were observed after day 7. Overall, both serum (Figure 2(a)) and vaginal wash (Figure 2(b)) Ig responses of rMOMP immunized mice were significantly higher $(P<0.05)$ than those of MOMP-278 immunized mice throughout the immunization time points.

Antibody isotyping studies were conducted to characterize the types of antibody (IgA, IgG1, IgG2a or IgG2b) induced in mice by immunization with rMOMP or rMOMP-278. To accomplish this, purified proteins (rMOMP or rMOMP-278) as well as UV-inactivated $C$. trachomatis Ebs were used as antigens in ELISA. Our results showed that i.m administration of rMOMP-278 and rMOMP, respectively to mice elicited up to 12 -fold and 40 -fold increase $(P<0.05)$ in the production of IgG1 antibodies in their serum by day 21 (Figure 3(a)) as compared to the day 0 or PBS group serum samples. When whole UV-inactivated C. trachomatis Ebs were used as antigen (Figure 3(b)) the responses were significantly less $(P<0.05)$; only a 4 -fold and 30 -fold increase in IgG1 antibodies were seen in serum of rMOMP-278 and rMOMP, respectively, suggesting the specificities of the elicited responses. Vaginal wash IgG1 responses were increased but at very low levels (ODs of $<0.1)$ for both the rMOMP and rMOMP-278 groups of mice (data not shown). Significant concentrations of serum IgA $(P<0.05)$ were induced in mice in response to rMOMP-278 and rMOMP immunizations by day 7 gradually increasing up to 6-fold and 11-fold, respectively by day 21 (Figure 4(a)). Again the serum IgA responses were lower when C. trachomatis Ebs were used; each immunogen induced 4-fold increases of IgA at day 21 (Figure 4(b)). We found that rMOMP-278 and rMOMP induced very high levels of IgA in vaginal wash samples as shown in Figure 4(c). The concentrations were markedly enhanced from 8- to 14-fold for rMOMP-278 and from 8- to 17-fold for rMOMP by day 21 (Figure 4(c)) as compared to day 0 and the PBS vaginal wash samples. Significant IgA antibodies in vaginal wash samples were not obtained when $C$. trachomatis Ebs were used as an- 


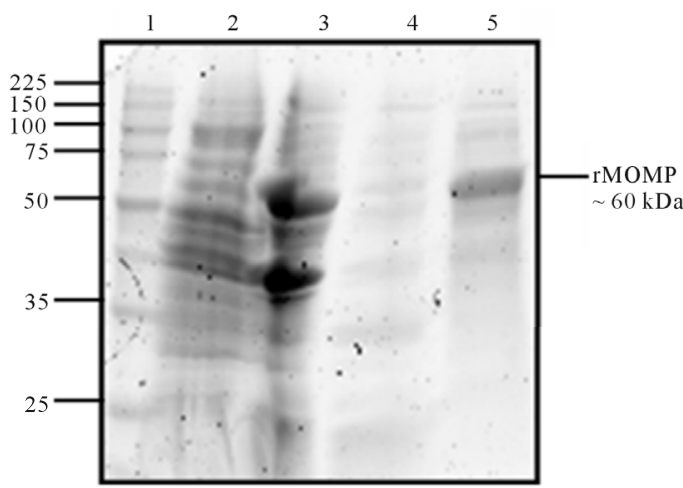

(a)

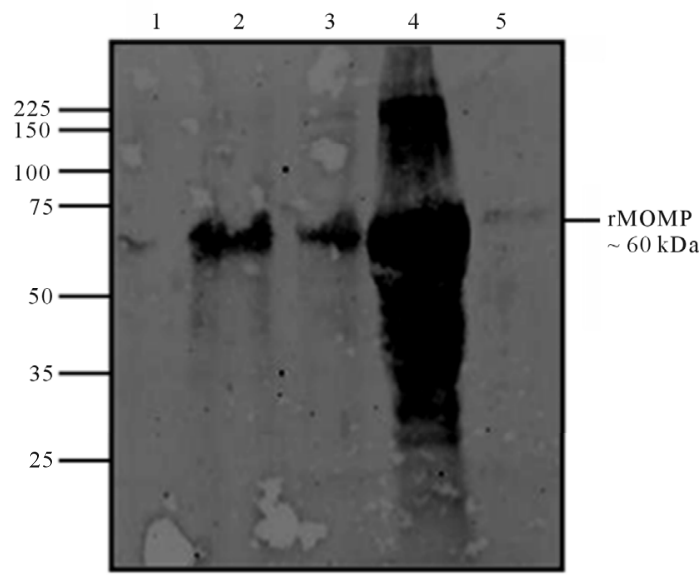

(c)

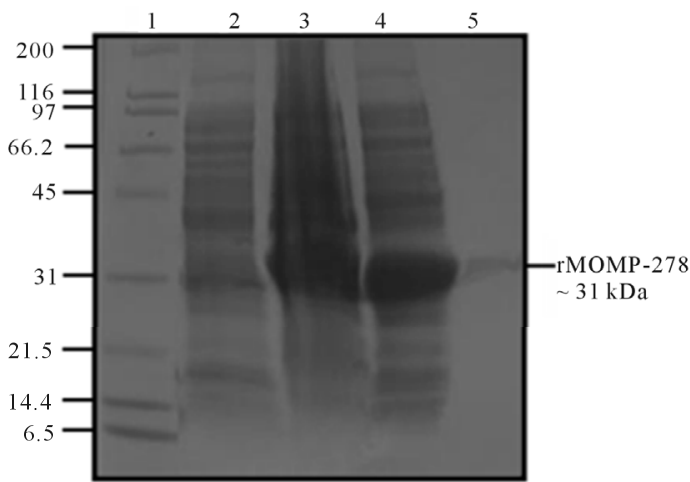

(b)

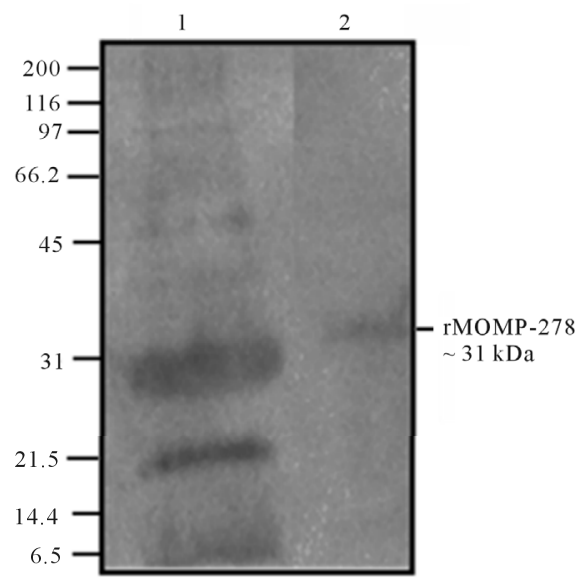

(d)

Figure 1. SDS-PAGE analyses of rMOMP and rMOMP-278. The rMOMP and rMOMP-278 clones were expressed and cytoplasmic extracts (CE) and inclusion bodies (pellet) were collected and analyzed. Inclusion bodies were solubilized (IB soluble) and purified on His-Bind columns (His purified). All samples were run on a $10 \%-20 \%$ linear gradient ready gel and stained with Coomassie blue (1\%). The rMOMP gel was designated as lane 1, MW; lane 2, IB soluble protein; lane 3, pellet; lane 4, CE; lane 5, purified rMOMP (a) while the gel for rMOMP-278 was designated as lane 1, MW; lane 2, CE; lane 3, pellet; lane 4, IB soluble protein; lane 5, purified rMOMP-278 (b). Purified rMOMP and rMOMP-278 were transferred onto a PVDF membrane and probed with anti-MOMP polyclonal antibody. Western blot analyses for rMOMP: lane 1, MW; lane 2, IB soluble protein; lane 3, CE; lane 4, pellet; lane 5, purified rMOMP (c). For rMOMP-278 blot: lane 1, IB soluble protein and lane 2, purified rMOMP (d).

tigen (data not shown).

Given that a $\mathrm{Th}_{1}$ antibody response is highly recommended in a Chlamydia vaccine candidate we next evaluated serum and vaginal wash $\operatorname{IgG} 2 \mathrm{a}\left(\mathrm{Th}_{1}\right.$ response) and $\mathrm{IgG} 2 \mathrm{~b}$ ( $\mathrm{Th}_{2}$ response) antibodies from immunized mice. In the serum of mice immunized with rMOMP-278, when using the rMOMP-278 as an antigen, the IgG2a response was similar to that in the serum of mice immunized with rMOMP and with rMOMP being used as an antigen (Figure 5(a)). In fact, the levels increased between 8- to 12-fold for both immunogens by the third immunizations. When using $C$. trachomatis as an antigen, the rMOMP-278 IgG2a responses were significantly lower than the $\operatorname{IgG} 2 \mathrm{a}$ response in groups administered
rMOMP (Figure 5(b)), and both of these responses were significantly lower than when using rMOMP or rMOMP278 as antigens.

The vaginal washes from mice immunized with rMOMP-278 did not contain significant amounts of $\operatorname{IgG} 2 \mathrm{a}$ antibodies irrespective of whether the antigen was rMOMP-278 or C. trachomatis Ebs. On the other hand only a 3 -fold increase in vaginal wash $\operatorname{IgG} 2$ a antibody response was seen in samples from rMOMP-immunized mice (data not shown). The IgG2b $\mathrm{Th}_{2}$ antibody was abundantly produced in sera from both groups immunized with rMOMP-278 (16- to 24-fold) and rMOMP (22- to 28-fold) (Figure 6(a)). In comparison an 8- to 12-fold and 20 - to 28 -fold increase in $\operatorname{IgG} 2 \mathrm{~b}$ antibodies were 


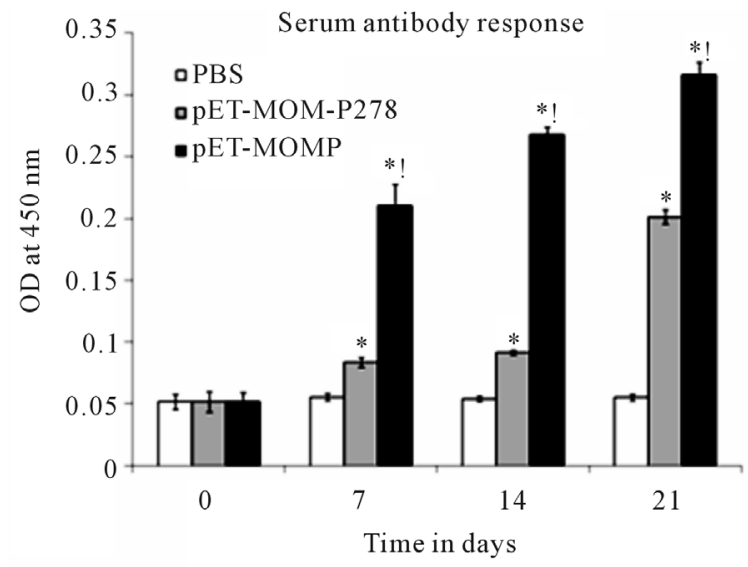

(a)

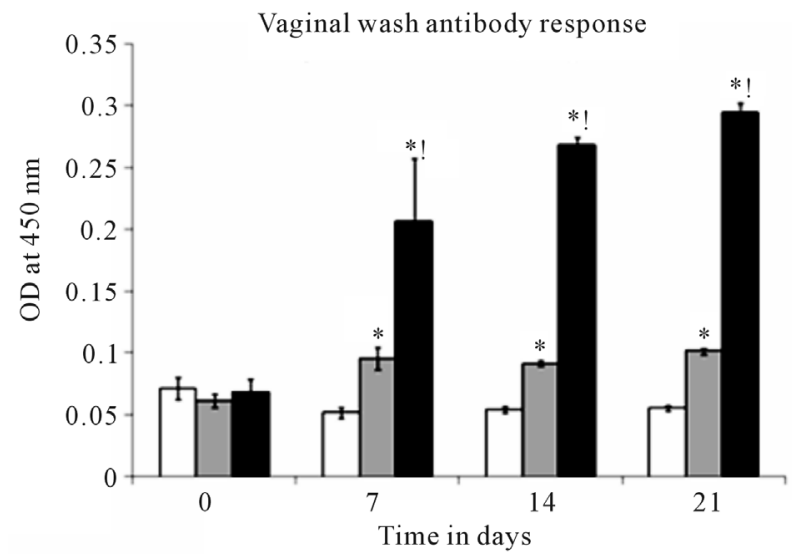

(b)

Figure 2. Serum (a) and vaginal wash (b) antibody responses induced in mice to rMOMP and rMOMP-278. Mice were immunized intramuscularly with either rMOMP, rMOMP-278 or PBS and serum and vaginal wash samples were collected at $0,7,14$ and 21 days post-immunization. rMOMP and rMOMP-278 specific total antibody responses were determined by ELISA using serum and vaginal wash samples at a 1:250 dilution. Results are presented as an average of data from groups of mice run in triplicate. Error bars represent standard deviation. *indicates significant differences exist between an individual immunogen and basal (PBS) responses at $P<0.05$. !indicates significant differences exist between rMOMP and rMOMP-278 at $P<$ 0.05 .

seen in rMOMP-278 and rMOMP immunized mice, respectively in response to $C$. trachomatis Ebs (Figure 6(b)). Vaginal wash IgG2b antibodies were undetected in mice from both immunized groups (data not shown).

\section{Discussion}

The development of a protective vaccine that induces long-term local genital mucosal immunity is paramount to preventing infection with $C$. trachomatis. However, despite decades of effort, to produce a vaccine against

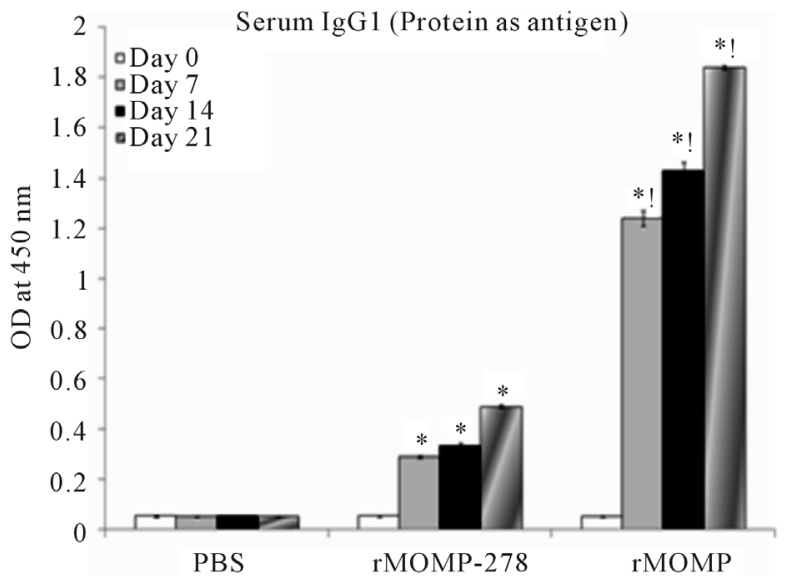

(a)

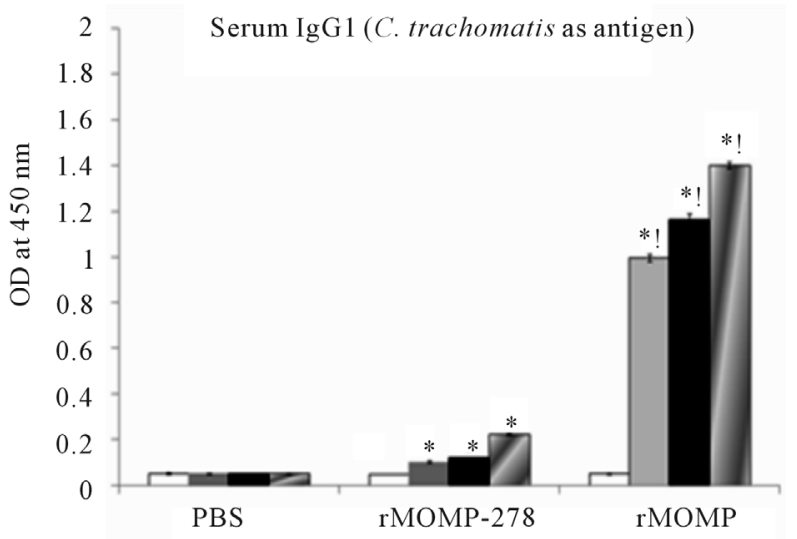

(b)

Figure 3. Serum IgG1 antibody response induced in mice to rMOMP and rMOMP-278. Mice were immunized intramuscularly with either rMOMP, rMOMP-278 or PBS and serum and vaginal wash samples were collected and analyzed as described in Figure 2 legend. The antigens used for the ELISA were rMOMP or rMOMP-278 (a) or $C$. trachomatis (b). Results are presented as an average of data from three groups run in triplicate. Statistics are as indicated in Figure 2 legend.

this pathogen, this goal has been futile with impediments seemingly substantial, such as the identifications of protective antigens, an effective adjuvant delivery system or a prominent $\mathrm{Th}_{1}$ mucosal and systemic antibody and cellular responses. Previously we showed that rMOMP genetically linked to modified cholera toxin was immunogenic in mice, with partial protection being afforded after a homologous challenge infection. Toward identifying T-cell epitopes that may be involved in this protective capacity, here in this report, we cloned a $31 \mathrm{kDa}$ peptide derivative of rMOMP (rMOMP-278) and tested its immunogenicity in mice. Our ultimate goal was to assess its potential as a vaccine candidate in mice, as compared to its parent rMOMP, in the absence of an adjuvant. The 


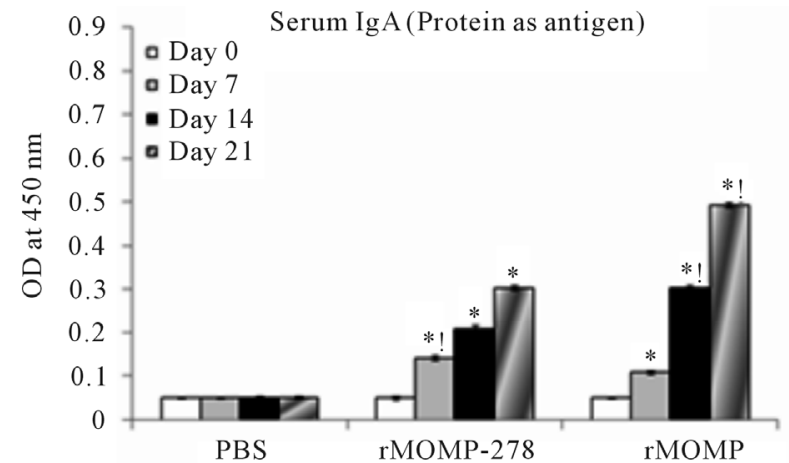

(a)

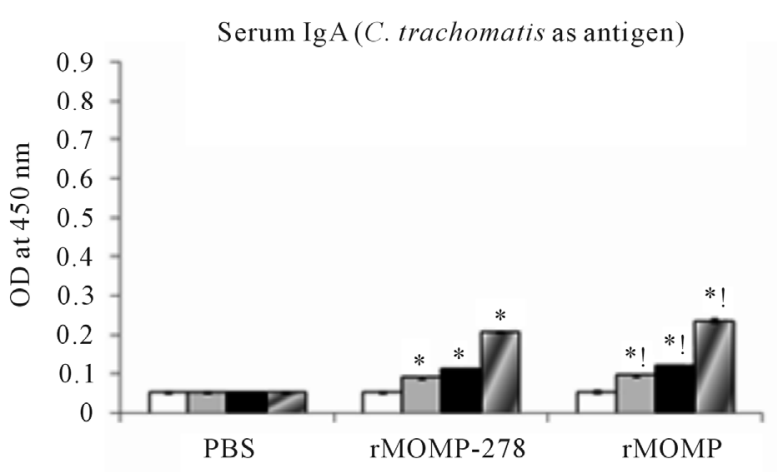

(b)

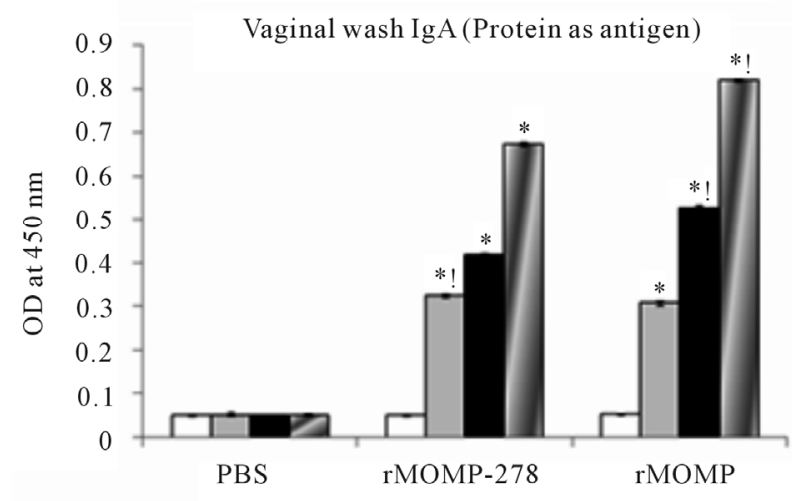

(c)

Figure 4. Serum and vaginal wash IgA antibody responses to rMOMP and rMOMP-278. Mice were immunized intramuscularly with either rMOMP, rMOMP-278 or PBS and serum and vaginal wash samples were collected and analyzed as described in Figure 2 legend. The antigens used for the ELISA were rMOMP or rMOMP-278 $(\mathrm{a}, \mathrm{c})$ or $C$. trachomatis (b). Results are presented as an average of data from three groups run in triplicate. Statistics are as indicated in Figure 2 legend.

findings from this study clearly demonstrate that the intramuscular administration of rMOMP-278 in the absence of an adjuvant can induce significant mucosal and systemic antibody responses with a $\mathrm{Th}_{1}$ bias, in some instances, being as robust as those induced by rMOMP in

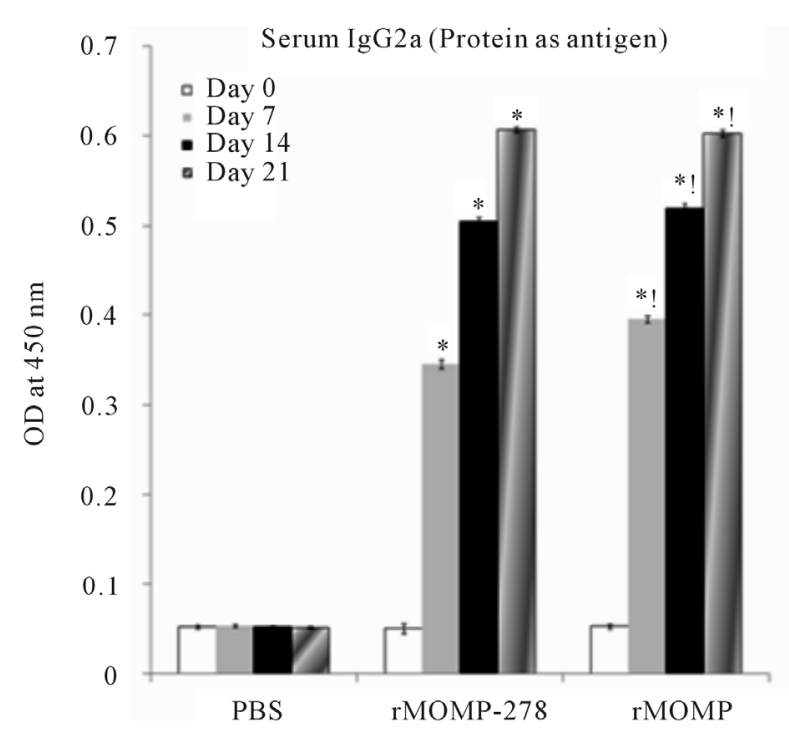

(a)

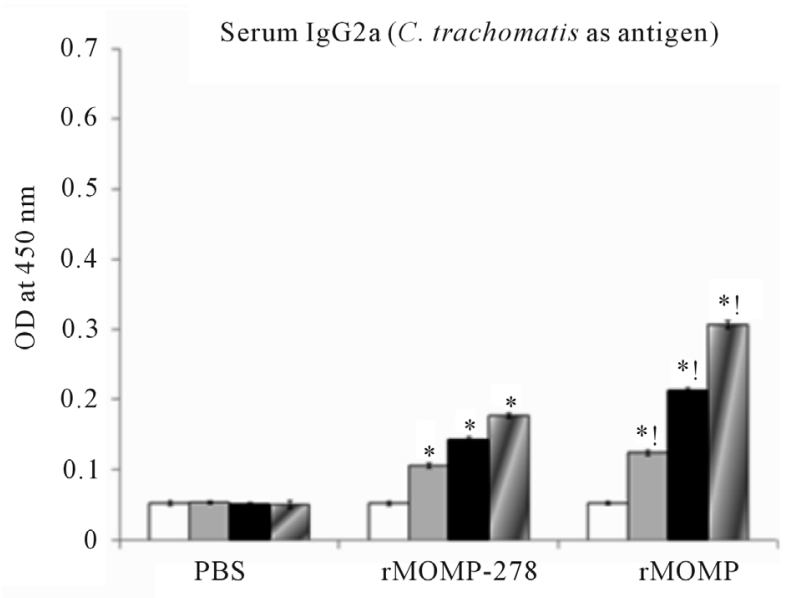

(b)

Figure 5. Serum IgG2a antibody responses to rMOMP and rMOMP-278. Mice were immunized intramuscularly with either rMOMP, rMOMP-278 or PBS and serum and vaginal wash samples were collected and analyzed as described in Figure 2 legend. The antigens used for the ELISA were rMOMP or rMOMP-278 (a) or C. trachomatis (b). Results are presented as an average of data from three groups run in triplicate. Statistics are as indicated in Figure 2 legend.

mice.

The present study shows that rMOMP-278 induced both total serum and vaginal wash antibody responses which were dependent on the numbers of immunizations given to mice. The levels of induction of these antibody responses, although significant as compared to the PBS group, were lower than those induced by the rMOMP. Moreover, three immunizations were required to produce heightened total antibody responses to rMOMP-278, whereas only a single immunization induced significant 


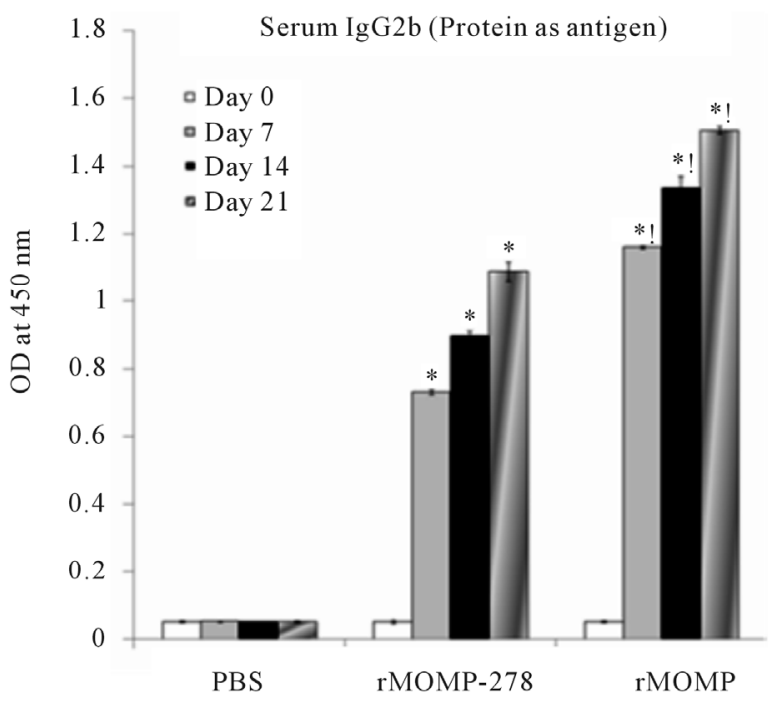

(a)

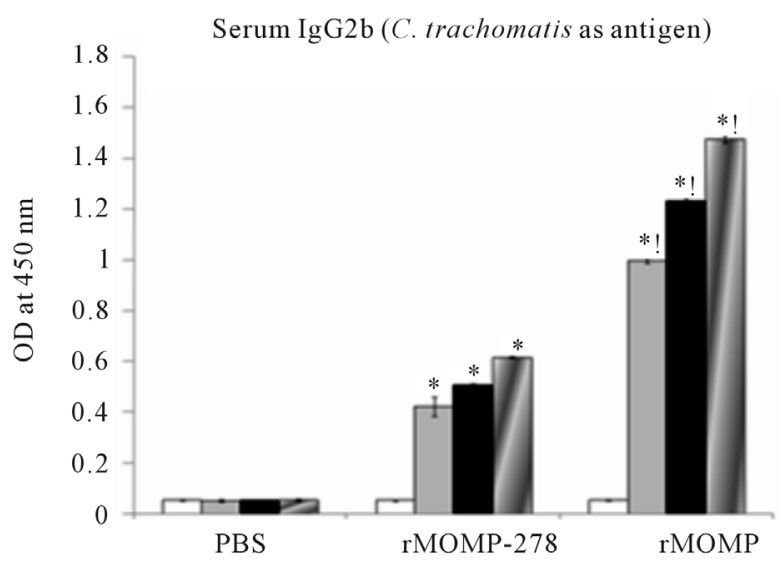

(b)

Figure 6. Serum IgG2b antibody responses to rMOMP and rMOMP-278. Mice were immunized intramuscularly with either rMOMP, rMOMP-278 or PBS and serum and vaginal wash samples were collected and analyzed as described in Figure 2 legend. The antigens used for the ELISA were rMOMP or rMOMP-278 (a) or C. trachomatis (b). Results are presented as an average of data from three groups run in triplicate. Statistics are as indicated in Figure 2 legend.

levels of antibodies to the rMOMP, suggesting that rMOMP with multiple epitopes are more effective in eliciting these antibody responses than its single peptide derivative. Although rMOMP induced higher responses than the rMOMP-278, both of these responses were lower than previously observed with rMOMP linked to modified cholera toxin [8] and rightfully so since an adjuvant was not used in the present study. Of no surprise, serum antibody responses were lower than vaginal wash antibody responses and independent of the route of im- munization since similar trends have been observed using the intranasal route [8], suggesting that the intramuscular route is as effective as the intranasal route in the induction of total antibody responses.

Further assessments of isotype antibodies of the total antibody responses revealed that rMOMP-278 induced both $\mathrm{Th}_{1}$ (IgG2a) and $\mathrm{Th}_{2}$ (IgG2b and IgG1) specific antibody isotypes in mice. Of significance was the induced responses were higher to the respective immunogens (rMOMP and rMOMP-278) than when the UVinactivated $C$. trachomatis Ebs were used in ELISAs, thus indicating the specificity of the elicited responses. The level of antigen-specific antibodies were detected in the order of $\operatorname{IgG} 2 b>\operatorname{IgG} 2 \mathrm{a}>\mathrm{IgG} 1$ for rMOMP-278 immunized mice. The pattern of antibody production differed when full MOMP was used as the immunogen, eliciting responses in the order of $\operatorname{IgG} 1>\operatorname{IgG} 2 \mathrm{~b}>\mathrm{IgG} 2 \mathrm{a}$. Apparently, rMOMP-278 induced more of a balanced $\mathrm{Th}_{1} / \mathrm{Th}_{2}$ response whereas the rMOMP induced more of a predominant $\mathrm{Th}_{2}$ response in the absence of an adjuvant. An interesting observation was that rMOMP-278 and rMOMP induced similar levels of the $\mathrm{Th}_{1} \operatorname{IgG} 2 \mathrm{a}$ antibody in both serum and vaginal wash samples in mice which is vital for Chlamydia protective immunity. Indeed, Chlamydia vaccine development has been a major hurdle since it is essential that the candidate immunogen must be able to induce a strong mucosal immune response along with $\mathrm{Th}_{1}$ and systemic antibody response [3]. CD4+ and CD8+ cells producing IFN- $\gamma$, as well as B cells are required to clear infection and prevent reinfection [3] CD4+ cells regulate B-cell production of neutralizing antibodies, which are important in preventing reinfection [22]. A recent study [23] highlighted that CD4+ $\mathrm{T}$ cells along with antibody contribute significantly to protective immunity during a Chlamydia infection as evidenced by the absence of protective immunity in vaccinated B-cell deficient mice challenged with $C$. trachomatis. We show that rMOMP-278 can, in the absence of an adjuvant, induce $\mathrm{Th}_{1}$ antibodies in mice.

Along with serum neutralizing antibodies, which are essential for protection, the most important antibody isotype in mucosal secretions that functions to provide protective immunity at mucosal surfaces is IgA [7]. Secretory $\operatorname{IgA}$ antibodies which are secreted by B cells in the mucosa are resistant to proteolytic cleavage in the protease rich milieu of genital tract and other mucosa sites $[3,7,22]$. Secretory IgA antibodies have also been shown to protect from chlamydial infections in mice [7,24-27] and humans [28]. Induction of mucosal $\operatorname{IgA}$ antibodies that protect from chlamydial infections is therefore of prime significance in its vaccine development regimen as recently demonstrated [14]. Our data shows that rMOMP- 
278 effectively targets genital tract mucosa by its ability to induce IgA in vaginal wash samples from mice, eliciting robust IgA responses similar to its parent rMOMP. Likewise, in terms of concentrations, the levels of serum and vaginal wash IgA induced by rMOMP-278 and rMOMP in mice were similar. The ability of the rMOMP-278 to induce similar levels of the $\mathrm{Th}_{1}$ (IgG2a) and of serum and mucosal $\operatorname{IgA}$ in mice as compared to its parent rMOMP strongly suggest that this peptide is most likely involved in the protection afforded by rMOMP conjugated to modified CTB [8] or to other vaccine delivery systems $[14,29]$. Both enhanced IgG2a and mucosal IgA have been correlated with protective immunity in vaccinated and $C$. trachomatis challenged mice [30].

Overall, the ability of rMOMP-278 to induce robust $\mathrm{Th}_{1}(\mathrm{IgG} 2 \mathrm{a})$ and $\operatorname{IgA}$ mucosal immune responses in mice provides compelling evidence that this peptide alone does have the properties of an immunogen which are highly desirable for a vaccine against $C$. trachomatis infection. Moreover, our data further suggest that rMOMP278 may contribute to protective immunity as elicited by full MOMP in mice as previously reported by us [8]. Ongoing studies to test the effectiveness of rMOMP-278 combined with a nanoparticle delivery system in mice are underway.

\section{Acknowledgements}

The project described was supported by NSF-CREST grant HRD-0734232.

\section{REFERENCES}

[1] J. U. Igietseme, C. M. Black and H. D. Caldwell, "Chlamydia Vaccines: Strategies and Status," Biology Drugs, Vol. 16, No. 1, 2002, pp. 19-35. doi:10.2165/00063030-200216010-00003

[2] F. O. Eko, Q. He, T. Brown, L. McMillan, G. O. Ifere, G. A. Ananaba, D. Lyn, W. Lubitz, K. L. Kellar, C. M. Black and J. U. Igietseme, "A Novel Recombinant Multisubunit Vaccine against Chlamydia," Journal of Immunology, Vol. 173, 2004, pp. 3375-3382.

[3] R. C. Brunham and J. Rey-Ladino, "Immunology of Chlamydia Infection: Implications for a Chlamydia trachomatis Vaccine," Nature Reviews Immunology, Vol. 5, No. 2, 2005, pp. 149-161. doi:10.1038/nri1551

[4] M. Tuffrey, I. Alexander, W. Conlan, C. Woods and M. Ward, "Hetrotypic Protection of Mice against Chlamydial Salpingitis and Colonization of the Lower Genital Tract with a Human Serovar F Isolate of $C$. trachomatis by Prior Immunization with Recombinant Serovar L1 Major Outer Membrane Protein," Journal of General Microbiology, Vol. 138, 1992, pp. 1707-1715.

[5] B. E. Batteiger, R. G. Rank, P. M. Bavoil and L. S. F. Soderberg, "Partial Protection against Genital Reinfection by Immunization of Guinea-Pigs with Isolated OuterMembrane Proteins of the Chlamydial Agent of GuineaPig Inclusion Conjunctivitis," Journal of General Microbiology, Vol. 139, 1993, pp. 2965-2972.

[6] S. C. Knight, S. Iqball, C. Woods, A. Stagg, M. E. Ward and M. Tuffrey, "A Peptide of Chlamydia trachomatis Shown to Be a Primary T-Cell Epitope in Vitro Induces Cell-Mediated Immunity in Vivo," Immunology, Vol. 85, 1995, pp. 8-15.

[7] H. Su, M. Parnell and H. D. Caldwell, "Protective Efficacy of a Parenterally Administered MOMP-Derived Synthetic Oligopeptide Vaccine in a Murine Model of Chlamydia trachomatis Genital Tract Infection: Serum Neutralizing IgG Antibodies Do Not Protect against Genital Tract Infection," Vaccine, Vol. 13, No. 11, 1995, pp. 1023-1032. doi:10.1016/0264-410X(95)00017-U

[8] S. R. Singh, K. Hulett, S. R. Pillai, V. A. Dennis, M. K. $\mathrm{Oh}$ and K. S. Gunn, "Mucosal Immunization with Recombinant MOMP Genetically Linked with Modified Cholera Toxin Confers Protection against Chlamydia trachomatis Infection," Vaccine, Vol. 24, No. 8, 2006, pp. 1213-1224. doi:10.1016/j.vaccine.2005.08.097

[9] J. Hansen, K. T. Jensen, F. Follmann, E. M. Agger, M. Theisen and P. Andersen, "Liposome Delivery of Chlamydia muridarum Major Outer Membrane Protein Primes a $\mathrm{Th}_{1}$ Response That Protects against Genital Chlamydial Infection in a Mouse Model," Journal of Infectious Diseases, Vol. 198, No. 5, 2008, pp. 758-767. doi: $10.1086 / 590670$

[10] C. Cheng, I. Bettahi, M. I. Cruz-Fisher, S. Pal, P. Jain, Z. Jia, J. Holmgren, A. M. Harandi and L. M. de la Mazaa, "Induction of Protective Immunity by Vaccination against Chlamydia trachomatis Using the Major Outer Membrane Protein Adjuvanted with CpG Oligodeoxynucleotide Coupled to the Nontoxic B Subunit of Cholera Toxin," Vaccine, Vol. 27, No. 44, 2009, pp. 6239-6246. doi:10.1016/j.vaccine.2009.07.108

[11] D. K. Hickey, F. E. Aldwell and K. W. Beagley, "Transcutaneous Immunization with a Novel Lipid-Based Adjuvant Protects against Chlamydia Genital and Respiratory Infections," Vaccine, Vol. 27, No. 44, 2009, pp. 6217-6225. doi:10.1016/j.vaccine.2009.08.001

[12] K. Laszlo, W. M. Whitmire, D. D. Crane, N. Reveneau, J. H. Carlson, M. M. Goheen, E. M. Peterson, S. Pal, L. M. de la Maza and H. D. Caldwell, "Chlamydia trachomatis Native Major Outer Membrane Protein Induces Partial Protection in Non-Human Primates: Implication for a Trachoma Transmission Blocking Vaccine," Journal of Immunology, Vol. 182, No. 12, 2009, pp. 8063-8070. doi:10.4049/jimmunol.0804375

[13] S. Guifeng, S. Pal, J. Weiland, E. M. Peterson and L. M. de la Maza, "Protection against an Intranasal Challenge by Vaccines Formulated with Native and Recombinant Preparations of the Chlamydia trachomatis Major Outer Membrane Protein," Vaccine, Vol. 27, No. 36, 2009, pp. 5020-5025. doi:10.1016/j.vaccine.2009.05.008

[14] H. Lü, H. Wang, H. M. Zhao, L. Zhao, Q. Chen, M. Qi, J. 
Liu, H. Yu, X. P. Yu, X. Yang and W. M. Zhao, "Dendritic Cells (DCs) Transfected with a Recombinant Adenovirus Carrying Chlamydial Major Outer Membrane Protein Antigen Elicit Protective Immune Responses against Genital Tract Challenge Infection," Biochemistry and Cell Biology, Vol. 88, No. 4, 2010, pp. 757-765. doi:10.1139/O10-011

[15] K. W. Beagley and P. Timms, "Chlamydia trachomatis Infection: Incidence, Health Costs and Prospects for Vaccine Development," Journal of Reproductive Immunology, Vol. 48, No. 1, 2000, pp. 47-68. doi:10.1016/S0165-0378(00)00069-3

[16] F. O. Eko, W. Lubitz, L. McMillan, K. Ramey, T. Moore, G. A. Ananaba, D. Lyn, C. M. Black and J. U. Igietseme, "Recombinant Vibrio cholerae Ghosts as a Delivery Vehicle for Vaccinating against Chlamydia trachomatis," Vaccine, Vol. 21, No. 15, 2003, pp. 1694-1703. doi:10.1016/S0264-410X(02)00677-1

[17] J. U. Igietseme and A. Murudin, "Induction of Protective Immunity against Chlamydia trachomatis Genital Infection by a Vaccine Based on Major Outer Membrane Protein-Lipophilic Imune Response-Stimulating Complexes," Infection and Immunity, Vol. 66, 2000, pp. 4030-4035.

[18] S. Kim and R. Demars, "Epitope Clusters in the Major Outer Membrane Protein of Chlamydia trachomatis," Current Opinion in Immunology, Vol. 13, No. 4, 2001, pp. 429-436. doi:10.1016/S0952-7915(00)00237-5

[19] L. Ortiz, M. Angevine, K. Suon-Kyeong, D. Watkins and R. Demars, "T-Cell Epitopes in Variable Segments of Chlamydia trachomatis Outer Membrane Protein Elicit Serovar-Specific Immune Responses in Infected Humans," Infection and Immunity, Vol. 68, No. 3, 2000, pp. 17191723. doi:10.1128/IAI.68.3.1719-1723.2000

[20] B. Toye, G. Zhong, R. Peeling and R. C. Brunham, "Immunologic Characterization of a Cloned Fragment Containing the Species-Specific Epitope from the Major Outer Membrane Protein of Chlamydia trachomatis," Infection and Immunity, Vol. 58, 1990, pp. 3909-3913.

[21] G. Zhong and R. C. Brunham, "Immunoaccessible Peptide Sequences of the Major Outer Membrane Protein from Chlamydia trachomatis Serovar C," Infection and Immunity, Vol. 58, 1990, pp. 3438-3441.

[22] W. P. Loomis and M. N. Starnbach, "T Cell Responses to Chlamydia trachomatis," Current Opinion in Microbiology, Vol. 5, No. 1, 2002, pp. 87-91. doi:10.1016/S1369-5274(02)00291-6

[23] C. M. Farris, S. G. Morrison and R. P. Morrison, "CD4+ T Cells and Antibody Are Required for Optimal MOMP Vaccine Induced Immunity to Chlamydia muridarum
Genital Infection," Infection and Immunity, Vol. 78, No. 10, 2010, pp. 4374-4383. doi:10.1128/IAI.00622-10

[24] S. Pal, I. Theodor, E. Peterson and L. M. de la Maza, "Immunization with the Chlamydia trachomatis Mouse Pneumonitis Major Outer Membrane Protein Can Elicit a Protective Immune Response against a Genital Challenge," Infection and Immunity, Vol. 69, No. 10, 2001, pp. 6240-6247. doi:10.1128/IAI.69.10.6240-6247.2001

[25] R. A. Hawkins, R. G. Rank and K. A. Kelly, "A Chlamydia trachomatis-Specific $\mathrm{Th}_{2}$ Clone Does Not Provide Protection against a Genital Infection and Displays Reduced Trafficking to the Infected Genital Mucosa," Infection and Immunity, Vol. 70, No. 9, 2002, pp. 5132-5139. doi:10.1128/IAI.70.9.5132-5139.2002

[26] L. J. Berry, D. K. Hickey, K. A. Skelding, S. Bao, A. M. Rendina, P. M. Hansbro, C. M. Gockel, K. W. Beagley, "Transcutaneous Immunization with Combined Cholera Toxin and $\mathrm{CpG}$ Adjuvant Protects against Chlamydia muridarum Genital Tract Infection," Infection and Immunity, Vol. 72, No. 2, 2004, pp. 1019-1028. doi:10.1128/IAI.72.2.1019-1028.2004

[27] D. K. Hickey, R. C. Jones, S. Bao, A. E. Blake, K. A. Skelding. L. J. Berry and K. W. Beagley, "Intranasal Immunization with $C$. muridarum Major Outer Membrane Protein (MOMP) and Cholera Toxin Elicits Local Production of Neutralising IgA in the Prostate," Vaccine, Vol. 22, No. 31-32, 2004, pp. 4306-4315. doi:10.1016/j.vaccine.2004.04.021

[28] R. C. Brunham, C. C. Kuo, L. Cles and K. K. Holmes, "Correlation of Host Immune Response with Quantitative Recovery of Chlamydia trachomatis from the Human Endocervix," Infection and Immunity, Vol. 39, 1983, pp. 1491-1494.

[29] K. A. Cunningham, A. J. Carey, L. Hafner, P. Timms and K. W. Beagley, "Chlamydia muridarum Major Outer Membrane Protein-Specific Antibodies Inhibit in Vitro Infection but Enhance Pathology in Vivo," American Journal of Reproductive Immunology, Vol. 65, No. 2, 2010, pp. 118-126. doi:10.1111/j.1600-0897.2010.00894.x

[30] A. K. Murthy, P. J. Chambers, P. A. Meier, G. Zhong and B. P. Arulanandam, "Intranasal Vaccination with a Secreted Chlamydial Protein Enhances Resolution of Genital Chlamydia muridarum Infection, Protects against Oviduct Pathology, and Is Highly Dependent upon Endogenous Gamma Interferon Production," Infection and Immunity, Vol. 75, No. 1, 2007, pp. 666-676. doi:10.1128/IAI.01280-06 